\title{
TWISTOR SPACES WITH MEROMORPHIC FUNCTIONS
}

\author{
Y. S. POON
}

(Communicated by Jonathan M. Rosenberg)

\begin{abstract}
Among the class of Kähler surfaces with zero scalar curvature, only the twistor space of those surfaces which are also Ricci-flat can admit nonconstant meromorphic functions. Moreover, the transcendental degree of the function field of the twistor space over such surfaces is equal to one. This result leads to a classification of twistor spaces with positive algebraic dimensions.
\end{abstract}

Introduction. In this article, we study the class of four dimensional compact connected Riemannian manifolds with a fixed orientation. The manifold $X$ is also assumed to be self-dual with constant scalar curvature $[3,18]$. Then the twistor space $Z$ over $X$ is the space of almost complex structure on $X$ compatible with the metric such that its canonical orientation is conjugate to the one given on $X$. As $X$ is assumed to be self-dual, the tautological almost complex structure on $Z$ is integrable [2]. In other words, $Z$ is a compact complex manifold.

On a complex manifold, one of its roughest birational invariants is its algebraic dimension $a(Z)$; i.e., the transcendental degree of its field of meromorphic functions over $\mathbf{C}$. It was shown by the author that if $a(Z)$ is equal to three then the self-dual conformal class on $X$ contains a metric with positive scalar curvature. However, when one allows the algebraic dimension simply to be nonnegative, $X$ can be a Ricci-flat surface as the K3-surface with Calabi-Yau metric and orientation conjugate to the canonical one. As a Kähler surface with zero scalar curvature is necessarily anti-self-dual [6], we shall consider Ricci-flat Kähler surfaces as a special subclass in the family of Kähler surfaces with zero scalar curvature and prove the following:

Theorem. Suppose that $X$, with its conjugate orientation, is a Kähler surface with zero scalar curvature. Then $a(Z) \leq 1$; with equality if and only if $X$ is Ricci-flat.

Received by the editors November 4, 1988 .

1980 Mathematics Subject Classification (1985 Revision). Primary 32L25; Secondary 14J30, $53 \mathrm{C} 55$.

The author was partially supported by NSF grant no. DMS- 8906806 . 
With this result and a result of Ville, one can also derive the following Corollary. If $X$ is simply-connected and $a(Z)$ is positive, then

(1) $X$ is either the K3-surface with the Calabi-Yau metric and conjugate orientation

or

(2) homeomorphic to the four sphere or the connected-sums of complex projective plane with positive scalar curvature.

This observation is an extension of Hitchin's work on Kählerian twistor spaces [13], the author's work on Moishézon twistor spaces [17] and is mainly motivated by the results of Ville [19] and the large class of manifolds that admit self-dual metrics [8]. We shall discuss this corollary and Ville's work toward the end of this article.

Proof of theorem. Suppose that $X$ is given as in the theorem. Let $J$ denote the complex structure on $X$, then $J$ and $-J$ are sections of $Z$ over $X$. The fact that they are also Kähler structures implies that they are parallel with respect to the induced connection on $Z$. Therefore, the images of $X$ via $J$ and $-J$ are horizontal holomorphic submanifolds in $Z$. They are denoted by $S$ and $\bar{S}$ respectively. The notation $\bar{S}$ is justified by the fact that the map $J_{x} \mapsto-J_{x}$ for any $J_{x}$ in a fibre of $Z$ over $X$ is an antiholomorphic involution. In fact, this involution is usually called the real structure [1]. This structure will play a vital role in our computation.

Since the associated bundle $\mathbf{S} \overline{\mathbf{S}}$ of the divisor $S+\bar{S}$ is invariant with respect to the real structure, so is its first Chern class. As the second cohomology group on $Z$ is generated by $c_{1}\left(\mathbf{K}^{-1}\right)$ and $H^{2}(X, \mathbf{R})$, where $\mathbf{K}^{-1}$ is the anticanonical bundle on $Z$, and the real structure acts on $H^{2}(X, \mathbf{R})$ by multiplication of $-1[13,16]$, the first Chern class of $\mathbf{S} \overline{\mathbf{S}}$ is a multiple of $c_{1}\left(\mathbf{K}^{-1}\right)$. Counting the intersection number with a generic fibre of $Z$ over $X$, we have

$$
c_{1}(\mathbf{S} \overline{\mathbf{S}})=1 / 2 c_{1}\left(\mathbf{K}^{-1}\right) \text {. }
$$

Note that there is a real holomorphic line bundle $\mathbf{K}^{-1 / 2}$ such that $\left(\mathbf{K}^{-1 / 2}\right)^{2}$ is isomorphic to $\mathbf{K}^{-1}$ [13], therefore, there is a real holomorphic line bundle $\mathbf{L}$ on $Z$ such that

$$
\mathbf{K}^{-1 / 2}=\mathbf{S} \overline{\mathbf{S}} \mathbf{L}
$$

with $c_{1}(\mathbf{L})$ vanishing. By the adjunction formula [10], one can check that the restriction of $\mathbf{S}$ onto $S$ is simply the anticanonical bundle of $S, \mathbf{K}_{S}^{-1}$. Since the divisors $S$ and $\bar{S}$ are disjoint, the isomorphism (1-1) is restricted onto $S$ and $\bar{S}$ to be

$$
\mathbf{K}_{\mid S}^{-1 / 2}=\mathbf{K}_{S}^{-1} \mathbf{L}_{\mid S} \text { and } \mathbf{K}_{\mid \bar{S}}^{-1 / 2}=\mathbf{K}_{\bar{S}}^{-1} \mathbf{L}_{\mid \bar{S}}
$$

Then for any holomorphic line bundle $\mathbf{F}$ on $Z$, we have the following exact sequences of sheaves of germs of sections:

$$
0 \rightarrow \mathscr{K}^{-m / 2} \mathscr{F} \mathscr{S}^{-1} \rightarrow \mathscr{K}^{-m / 2} \mathscr{F} \rightarrow \mathscr{O}_{S}\left(\mathbf{K}_{S}^{-m} \mathbf{L}^{-m} \mathbf{F}\right) \rightarrow 0
$$




$$
0 \rightarrow \mathscr{K}^{-(m-1) / 2} \mathscr{L} \mathscr{F} \rightarrow \mathscr{K}^{-m / 2} \mathscr{F} \mathscr{S}^{-1} \rightarrow \mathscr{O}_{\bar{S}}\left(\mathbf{K}_{\bar{S}}^{-m} \mathbf{L}^{-m} \mathbf{F}\right) \rightarrow 0 .
$$

Their induced exact sequences of cohomology yield an injection:

$$
0 \rightarrow H^{0}\left(Z, \mathscr{K}^{-(m-1) / 2} \mathscr{L} \mathscr{F}\right) \rightarrow H^{0}\left(Z, \mathscr{K}^{-m / 2} \mathscr{F}\right) .
$$

If $X$ is not Ricci-flat and $c_{1}(\mathbf{F})=0$, this injection is an isomorphism. To prove this assertion, we simply have to check that

$$
h^{0}\left(S, \mathscr{K}_{S}^{-m} \mathscr{L}^{-m} \mathscr{F}\right)=0 \text { and } h^{0}\left(\bar{S}, \mathscr{K}_{\bar{S}}^{-m} \mathscr{L}^{-m} \mathscr{F}\right)=0 \text {, for all } m \neq 0 .
$$

This is essentially a vanishing theorem of Yau [20]. The reason is that when $X$ is not Ricci-flat, $c_{1}\left(\mathbf{K}_{X}\right)$ is not equal to zero, neither is $c_{1}\left(\mathbf{K}_{S}^{-1}\right)$. In particular, the first Chern class of $\mathbf{K}_{S}^{-m} \mathbf{L}^{-m} \mathbf{F}$ is not equal to zero and hence it is a nontrivial bundle on the surface $S$. If it had a nontrivial holomorphic section, the set of zeros forms an effective divisor $D$. As the Kähler metric on $X$ is also the Kähler metric on $S$, the volume of $D$ in $S$ is equal to the cup product of $m c_{1}\left(\mathbf{K}_{S}^{-1}\right)$ with the Kähler class. On the other hand, this cup product is exactly the total scalar curvature and therefore is equal to zero in our case. Then the volume of $D$ would have been equal to zero. This contradiction shows that $\mathbf{K}_{S}^{-m} \mathbf{L}^{-m} \mathbf{F}$ has no nontrivial sections on $S$. The same argument works on $\bar{S}$. Therefore, the injection (1-5) is an isomorphism. Inductively, it gives

$$
H^{0}\left(Z, \mathscr{L}^{m} \mathscr{F}\right) \cong H^{0}\left(Z, \mathscr{K}^{-m / 2} \mathscr{F}\right) .
$$

But the first Chern class of $\mathbf{L}^{m} \mathbf{F}$ is equal to zero, Lemma 2.1 of [16] shows that this line bundle cannot have nonzero sections except when it is the trivial bundle. However, when it is the trivial bundle, then

$$
\mathbf{K}^{-m / 2} \mathbf{F}=(\mathbf{S} \overline{\mathbf{S}})^{m} \mathbf{L}^{m} \mathbf{F}=(\mathbf{S} \overline{\mathbf{S}})^{m} .
$$

The same lemma also requires $m$ to be positive when $\mathbf{K}^{-m / 2} \mathbf{F}$ has nonconstant sections. Therefore, under the assumption that $X$ is not Ricci-flat, for any $\mathbf{F}$ with $c_{1}(\mathbf{F})=0$, the bundle $\mathbf{K}^{-m / 2} \mathbf{F}$ has no sections except when it is isomorphic to $(\mathbf{S} \overline{\mathbf{S}})^{m}$ and $m \geq 0$, in which case the space of sections has only one dimension.

Since the argument of deriving (1-1) actually proves that any real line bundle $\mathbf{V}$ is isomorphic to $\mathbf{K}^{-m / 2} \mathbf{F}$ for some $m$ and some real $\mathbf{F}$ with vanishing first Chern class, then

$$
h^{0}(Z, \mathscr{V}) \leq 1 .
$$

Let us assume, to the contrary of the theorem, that the twistor space has a nonconstant meromorphic function when $X$ is not Ricci-flat. Let $D_{0}$ and $D_{\infty}$ be the divisors of zeros and poles respectively. They have isomorphic associated line bundles [10]. This line bundle is denoted by $\mathbf{D}$. Since $\mathbf{D} \overline{\mathbf{D}}$ is a real bundle with nontrivial sections, then the preceding argument shows that $\mathbf{D} \overline{\mathbf{D}}$ is isomorphic to $(\mathbf{S} \overline{\mathbf{S}})^{m}$ for some positive $m$. Moreover, the only divisor of this bundle is $m(S+\bar{S})$. Therefore, the divisors $D_{0}+\bar{D}_{0}$ and $D_{\infty}+\bar{D}_{\infty}$ are not only linearly equivalent but also identical . As we can assume that $D_{0}$ and $D_{\infty}$ 
have no common components, this identity implies that the conjugation of the divisor of poles is exactly the divisor of zeros. Therefore, the bundle $\mathbf{D}$ is real. It follows that this bundle has at most one divisor and hence the divisor of zeros would have been identical to the divisor of poles. This contradiction shows that any meromorphic function on the twistor space associated to a non-Ricci-flat Kähler surface with zero scalar curvature has to be constant.

To complete the proof of our theorem, we simply have to check that the algebraic dimension of the twistor space associated to a Ricci-flat Kähler surface is always equal to one. By a fundamental theorem of Cheeger and Gromoll [5], all such surfaces have the form $X / G$ where $G$ is a finite group of isometry on $X$ and $X$ is either the flat torus or a simply-connected compact Ricci-flat Kähler surface. In fact, if $X$ is not the torus, it is the K3-surface with the Calabi-Yau metric [5]. Therefore, the torus and the K3-surfaces are the universal models.

If $X$ is the torus or the K3-surface, their holonomy is contained in $S U(2)$. Then one can find a holomorphic submersion [11]:

$$
p: Z \rightarrow \mathbf{P}^{1}
$$

from the twistor space onto $\mathbf{P}^{1}$ such that $\mathbf{K}^{-1 / 2}=p^{*} \mathbf{H}^{2}$ where $\mathbf{H}$ is the hyperplane bundle on $\mathbf{P}^{1}$. The fibres of $p$ can be considered as a smooth copy of $X$ with a complex structure compatible with the Ricci-flat metric on $X$. In this description, it is obvious that $Z$ has nonconstant meromorphic functions. To check that $Z$ has algebraic dimension one, we may simply observe that a generic torus or K3-surface has algebraic dimension zero [4]. However, we prefer to give an argument in the line of our previous computation to show that the only line bundle on the twistor space that has nontrivial sections is the $p^{*} \mathbf{H}^{m}$.

Note that any conjugate pair of points on $\mathbf{P}^{1}$ determines a conjugate pair of divisors $S$ and $\bar{S}$ in $Z$, therefore,

$$
\mathbf{S} \overline{\mathbf{S}}=\mathbf{K}^{-1 / 2}
$$

This is a refinement of (1-1). Then for any line bundle $\mathbf{F}$ on $Z$, we have the exact sequences as the (1-3) and (1-4). However, in the present case, the canonical bundles of $S$ and $\bar{S}$ are both trivial as the canonical bundle of $X$ is. When $\mathbf{F}$ is a bundle with vanishing first Chern class and nontrivial on $S$, one can apply Yau's vanishing theorem again and prove that there is a natural isomorphism:

$$
H^{0}(Z, \mathbf{F}) \cong H^{0}\left(Z, \mathbf{K}^{-m / 2} \mathbf{F}\right)=0, \text { for all } m \geq 0 .
$$

As $\mathbf{F}$ has vanishing first Chern class, it has no global sections [16], and hence $\mathbf{K}^{-m / 2} \mathbf{F}$ has no nontrivial sections. In other words, if this bundle is real and has nontrivial global sections, it has to be trivial on every conjugate pair $S$ and $\bar{S}$. It follows that $\mathbf{F}=p^{*} \mathbf{H}^{k}$ for some $k$. But $F$ has vanishing first Chern class and therefore, $k=0$ and $\mathbf{F}$ is actually a trivial bundle. 
As any real line bundle has the form $\mathbf{K}^{-m / 2} \mathbf{F}$, the only real line bundles with nontrivial sections are $\mathbf{K}^{-m / 2}$ with $m \geq 0$. If $\mathbf{D}$ is any line bundle with effective divisor, then the reality of $\mathbf{D} \overline{\mathbf{D}}$ implies that

$$
\mathbf{D} \overline{\mathbf{D}}=\mathbf{K}^{-m / 2}=p^{*} \mathbf{H}^{2 m} .
$$

Since $D$ and $\bar{D}$ have the same intersection number with a real twistor line,

$$
\mathbf{D}=p^{*} \mathbf{H}^{m} \otimes \mathbf{L} \text { and } \overline{\mathbf{D}}=p^{*} \mathbf{H}^{m} \otimes \mathbf{L}^{-1},
$$

for some line bundle $\mathbf{L}$ that is trivial on every real twistor line. But the bundles $\mathbf{D}$ and $\overline{\mathbf{D}}$ have nontrivial sections, the argument in the last paragraph shows that the bundle $\mathbf{L}$ is trivial. Therefore,

$$
\mathbf{D}=\overline{\mathbf{D}}=p^{*} \mathbf{H}^{m} \text {. }
$$

It means that any meromorphic function on $Z$ is given by a composition of $p$ with a meromorphic function on $\mathbf{P}^{1}$ and hence $a(Z)=1$.

Suppose that $\tilde{X}=X / G$ is a general Ricci-flat Kähler surface and $G$ is a finite subgroup of isometry on $X$ which is a flat torus or a K3-surface, then the twistor space $\tilde{Z}$ of $\tilde{X}$ is given by $Z / G$ where $Z$ is the twistor space of $X . G$ acts on $Z$ because, in general, conformal transformation on $X$ induces holomorphic transformation on $Z$. To see the smooth action, recall that the projection $p$ is differentiably trivial. Therefore, as a smooth manifold $Z=X \times \mathbf{P}^{1}$. Then the action $G$ on $Z$ is the isometry on the factor $X$ and the following action on $\mathbf{P}^{1}$ :

$$
J \mapsto(d g) \circ J \circ(d g)^{-1}
$$

for $J$ in $\mathbf{P}^{1}$ as a compatible complex structure on $X$ and $g$ in the group $G$ as a map from the manifold $X$ onto itself. One may also take the viewpoint that the group $G$ acts on $Z$ and $\mathbf{P}^{1}$ holomorphically and then the holomorphic projection $p$ is $G$-equivariant.

Since $\tilde{Z}=Z / G$, the algebraic dimension of $\tilde{Z}$ is at most one. On the other hand, the action $G$ on $\mathbf{P}^{1}$ is actually a finite subgroup of the standard $S O(3)$ action on $\mathbf{P}^{1}$ because it is a subgroup of isometry on $X$. Moreover, as the action on each real twistor line commutes with the antipodal map, the $G$-action on $\mathbf{P}^{1}$ is a finite subgroup of the standard action of $S U(2)$. This type of group is classically called the binary polyhedral group. As there are $G$-invariant polynomials on $\mathbf{C}^{2}$ found by Klein [14], there are $G$-invariant meromorphic functions on $\mathbf{P}^{1}$. Therefore, the algebraic dimension of $\tilde{Z}$ is also equal to 1 . The proof of our theorem is completed.

Proof of corollary. In [19], Ville claimed that if twistor space has positive algebraic dimension, then the self-dual conformal class on $X$ contains a metric with nonnegative constant scalar curvature. As the proof over a simply-connected $X$ is fairly easy, we shall follow Ville's argument in this special case and prove the 
following:

Lemma (2-1) [17, 19]). If $a(Z)$ is positive and $X$ is simply connected, then the scalar curvature on $X$ is nonnegative.

If the scalar curvature is positive, there is no harmonic anti-self-dual 2-forms on $X$ and therefore, the intersection form on $X$ is positive definite [12]. It follows that $X$ is homeomorphic to the connected-sums of complex projective planes $[7,9]$. On the other hand,

Lemma (2-2). If $X$ is simply-connected with zero scalar curvature and $a(Z)$ is positive, then $X$, with its conjugate orientation, is a Kähler surface.

As a consequence of our theorem, $X$ is the K3-surface with Calabi-Yau metric and conjugate orientation.

To prove the above two lemmas, let us first observe that the simple connectivity of $X$ implies that a holomorphic line bundle on the twistor space is uniquely determined by its first Chern class. To check this claim, one simply has to show that the Hodge number $h^{0,1}$ is equal to zero. Recall that the Todd genus is the alternating sum: $h^{0,0}-h^{0,1}+h^{0,2}-h^{0,3}$. For a twistor space, it is equal to $1-b_{1}+b_{-}$[13], where $b_{-}$is the dimension of the space of anti-self-dual harmonic 2-forms. By Serre duality, $h^{0,3}$ is equal to the dimension of the space of sections of the canonical bundle which is always equal to zero [13]. By the twistor correspondence, $b_{-}$is equal to the dimension of $H^{1}(Z, \mathbf{K})$ [12]. Then Serre duality implies that $h^{0,2}=b_{-}$. Therefore, $b_{1}=h^{0,1}$. Hence when $X$ is simply connected, $h^{0,1}=0$.

Proof of Lemma (2-1). Suppose that the twistor space has positive algebraic dimension, there is at least one non-constant meromorphic function on $Z$. Then the divisors of zeros and poles are the divisors of a nontrivial holomorphic section of a holomorphic line bundle, say $\mathbf{F}$. Let $\mathbf{L}$ be $\mathbf{F} \overline{\mathbf{F}}$, then $\mathbf{L}$ is a real line bundle. It follows that the first Chern class of $\mathbf{L}$ is an integral multiple of the one of $\mathbf{K}^{-1 / 2}$ and hence $\mathbf{L}=\mathbf{K}^{-m / 2}$ for some integer $m$. As $\mathbf{F}$ has nontrivial divisors, so does $\mathbf{L}$. Then $m$ is strictly positive [13].

On the other hand, there is the twistor operator $\bar{D}_{m}$ defined by the composition of the induced connection $D$ on the bundle of $-1 / 2$-spinors with weight $m$ with an orthogonal projection $[2,12]$ :

$$
\bar{D}_{m}: S_{-}^{m} \rightarrow S_{-}^{m} \otimes S_{+} \otimes S_{-} \rightarrow S_{-}^{m+1} \otimes S_{+} .
$$

As $\left\|\bar{D}_{m} s\right\|^{2}=a\|D s\|^{2}-b u\|s\|^{2}$, where $a, b$ are positive universal constants depending on $m, u$ is the scalar curvature [17], when the scalar curvature is negative, $\bar{D}_{m}$ has no non-zero kernel.

However, the space of the solutions to the equation $\bar{D}_{2 m} s=0$ is naturally isomorphic to the space of holomorphic sections of $\mathbf{K}^{-m / 2}$ [13], therefore, when 
the scalar curvature is negative, this bundle has no holomorphic sections and hence the twistor space cannot have non-constant meromorphic functions.

Proof of Lemma (2-2). We have seen that if there are non-constant meromorphic functions on the twistor space, the bundle $\mathbf{K}^{-m / 2}$ has non-zero global sections, for some positive $m$. It implies that the equation $\bar{D}_{2 m} s=0$ has nontrivial sections. When the scalar curvature is equal to zero, the solutions to this equation are exactly the parallel sections of the bundle $S_{-}^{2 m}$. Then the structure group of this bundle is reduced from $S O(3)$ to $U(1)$ and the holonomy of the principle bundle of the frame on $X$ is reduced from $S U(2) \times{ }_{z_{2}} S U(2)$ to $S U(2) \times \mathrm{z}_{2} U(1)$, i.e., $U(2)$. It means that the metric on $X$ is a Kähler metric. However, the orientation induced by the complex structure is conjugate to the given one because the Kähler form which is a parallel section of $S_{-}^{2}$ is anti-self-dual. It completes the proof.

\section{ACKNOWLEDGMENT}

I thank M. Ville for telling me her result by which this piece of work is motivated. Also, I thank A. N. Todorov for many interesting conversations and N. J. Hitchin for his help.

\section{REFERENCES}

1. M. F. Atiyah, K-theory and reality, Quart. J. Math. Oxford (2) 17 (1966), 369-387.

2. M. F. Atiyah, N. J. Hitchin and I.M. Singer, Self-duality in four dimensional Riemannian geometry, Proc. Roy. Soc. London Ser. A 362 (1978), 425-461.

3. T. Aubin, The scalar curvature, Differential geometry and relativity (Cahen and Flato, eds.) 1976.

4. W. Barth, C. Peters and A. Van de Ven, Compact complex surfaces, Springer-Verlag, Berlin, 1984.

5. A. Besse, Einstein manifolds, Springer-Verlag, Berlin, 1987.

6. C.P. Boyer, Conformal duality and compact complex surfaces, Math. Ann 274 (1986), 517-526.

7. S. K. Donaldson, An application of gauge theory to the topology of 4-manifolds, J. Differential Geom. 18 (1983), 269-316.

8. S. K. Donaldson and R. Friedman, Connected sums of self-dual manifolds and deformations of singular spaces, Nonlinearity 2 (1989), 197-239.

9. M. Freedman, The topology of four-dimensional manifolds, J. Differential Geom. 17 (1982), 357-454.

10. P. Griffiths and J. Harris, Principles of algebraic geometry, Wiley, New York, 1978.

11. N. J. Hitchin, Polygons and gravitons, Math. Proc. Cambridge Phil. Soc. 85 (1979), 465-476.

12. __ Linear field equations on self-dual spaces, Proc. Roy. Soc. London Ser. A 370 (1980), 173-191.

13. __ Kählerian twistor spaces, Proc. London Math. Soc. 43 (1981), 173-191.

14. F. Klein, Vorlesung über das Ikosaeder und die Auflösung der Gleichung vom fünften Grade, Leibzeig, 1884.

15. S. Kobayashi and K. Nomizu, Foundation of differential geometry I, II, Wiley and Sons, New York, 1969. 
16. Y. S. Poon, Compact self-dual manifolds with positive scalar curvature, J. Differential Geom. 24 (1986), 97-132.

17. __ Algebraic dimension of twistor spaces, Math. Ann. 282 (1988), 621-627.

18. R. Schoen, Conformal deformation of a Riemannian metric to constant scalar curvature, J. Differential Geom. 20 (1984), 479-495.

19. M. Ville, On twistor spaces with positive algebraic dimension, preprint.

20. S.T. Yau, On the curvature of compact Hermitian manifolds, Invent. Math. 25 (1974), 213-239.

Department of Mathematics, Rice University, P.O. Box 1892, Houston, Texas 77251 